Pacific Journal of Mathematics

ON THE HEAT EQUATION FOR HARMONIC MAPS FROM
NONCOMPACT MANIFOLDS 


\title{
ON THE HEAT EQUATION FOR HARMONIC MAPS FROM NON-COMPACT MANIFOLDS
}

\author{
Guojun G. Liao and Luen-Fai Tam
}

\begin{abstract}
Harmonic maps are critical points of the energy functional for maps between Riemannian manifolds. In this paper we study the heat equation for harmonic maps from a non-compact manifold $M$ into $N$. We show that if the target manifold $N$ is compact and has non-positive sectional curvature, and if the initial map has finite total energy, then there exists a solution $u(x, t): M \times[0, \infty) \rightarrow N$ and a sequence $t_{j} \rightarrow \infty$, such that $u\left(\cdot, t_{j}\right)$ converges on compact subsets of $M$ to a harmonic from $M$ into $N$. We also obtain some basic properties of the solution $u(x, t)$. In particular, we prove a uniqueness theorem for the solution and a monotonicity theorem for the energy functional.

Eells and Sampson proved that if $(M, g)$ and $\left(N, g^{\prime}\right)$ are compact Riemannian manifolds, $\left(N, g^{\prime}\right)$ has non-positive sectional curvature, then any smooth map $h: M \rightarrow N$ is homotopic to a smooth harmonic map. They established the existence of a solution $u(x, t)$ : $M \times[0, \infty) \rightarrow N$, of $(1.1)$ in $\S 1$, and showed that there exists $t_{j} \rightarrow \infty$, such that $u\left(\cdot, t_{j}\right)$ converges to a smooth harmonic map from $M$ into $N$. Schoen and Yau showed that if $M$ is complete noncompact and if $h: M \rightarrow N$ has finite energy, then $h$ is homotopic on any compact subsets of $M$ to a harmonic map. Their method is based on Hamilton's results on harmonic maps from a manifold with boundary. By studying the heat equation directly, we recovered the result of Schoen and Yau. We believe the basic properties of solutions of the heat equation established in this paper will be useful in the study of harmonic maps on non-compact manifolds.
\end{abstract}

1. Existence. Let $\left(M^{m}, g\right)$ and $\left(N^{n}, g^{\prime}\right)$ be complete Riemannian manifolds. $M$ is non-compact. We want to study the initial value problem for the heat flow for harmonic maps. More precisely, we want to study the following system for a map $u: M \times[0, \infty) \rightarrow N$, in local coordinates $x=\left(x^{1}, \ldots, x^{m}\right)$, and $u=\left(u^{1}, \ldots, u^{n}\right)$ on $M$ and $N$ respectively:

$$
\left\{\begin{array}{l}
\Delta_{M} u^{\alpha}-\frac{\partial u^{\alpha}}{\partial t}=-g^{i j} \frac{\partial u^{\beta}}{\partial x^{i}} \frac{\partial u^{\gamma}}{\partial x^{j}} \Gamma_{\beta \gamma}^{\alpha}, \\
\quad \text { in } M \times(0, \infty), \alpha=1, \ldots, n ; \\
u(x, 0)=h(x),
\end{array}\right.
$$

where $\Delta_{M}$ is the Laplace-Beltrami operator on $M, \Gamma_{\beta \gamma}^{\alpha}$ are the 
Christoffel symbols on $N, \quad\left(g^{i j}\right)=\left(g_{i j}\right)^{-1}$ and $h \in C^{\infty}(M, N)$. We use the convention that Latin letters range from 1 to $m$, and Greek letters range from 1 to $n$. In this section, we want to prove the following:

THEOREM 1.1. Let $M$ be a complete non-compact Riemannian manifold. Suppose $N$ is compact without boundary with non-positive curvature. Then (1.1) has a solution for all $h(x)$ with finite total energy.

Recall that for a map $h:(M, g) \rightarrow\left(N, g^{\prime}\right)$, the energy density of $h$ is given by

$$
e(h)=g^{i j} \frac{\partial h^{\alpha}}{\partial x^{i}} \frac{\partial u^{\beta}}{\partial x^{j}} g_{\alpha \beta}^{\prime}
$$

where $x^{i}$, and $u^{\alpha}, 1 \leq i \leq m, 1 \leq \alpha \leq n$, are local coordinates of $M$ and $N$ respectively. The total energy is defined as

$$
E(h)=\int_{M} e(h) d V_{M} .
$$

Let $\left\{\Omega_{k}\right\}_{k=1}^{\infty}$ be a compact exhaustion of $M$ satisfying:

(i) $\Omega_{k} \subset \subset \Omega_{k+1}, k=1,2,3, \ldots$;

(ii) $\bigcup_{k=1}^{\infty} \Omega_{k}=M$;

(iii) $\partial \Omega_{k}$ is smooth, $k=1,2,3, \ldots$;

(iv) $\operatorname{diam}\left(\boldsymbol{\Omega}_{k}\right)<\operatorname{dist}\left(\boldsymbol{\Omega}_{k}, \partial \boldsymbol{\Omega}_{k+1}\right)$.

Hence for $x, y \in \Omega_{k}$, any minimal geodesic joining $x$ any $y$ must lie inside $\Omega_{k+1}$. In order to apply some results of heat kernels in [C-L-Y], for each $k$ we construct a complete manifold $\left(M_{k}, g_{k}\right)$ so that

(i) $\Omega_{k} \subset M_{k}$, and in $\Omega_{k}, g_{k}=g$;

(ii) the complement of a compact neighborhood of $\Omega_{k}$ in $M_{k}$ is isometric to $\partial \Omega_{k} \times[0, \infty)$.

This can be done by considering the exponential map on the normal bundle of $\partial \Omega_{k}$. Note that the curvature tensor of $M_{k}$ and its covariant derivatives are uniformly bounded, and the injectivity radius of $M_{k}$ is also bounded away from 0 . The following result is from [C-L-Y]:

LEMMA 1.2. For any $T>0$, there exists a constant $C_{1}>0$ depending on $M_{k}$ and $T$ and another constant $C_{2}>0$ depending only on $m$, such that if $H_{k}(x, y, t)$ is the heat kernel of $M_{k}$ and if $\left|D_{l} H_{k}\right|$ 
denotes the norm of the lth covariant derivatives of $H_{k}(x, y, t)$, then

$$
\left|D_{l} H_{k}\right|(x, y, t) \leq C_{1} t^{-\left(\frac{m+l}{2}\right)} \exp \left(-\frac{C_{2} r_{k}^{2}(x, y)}{t}\right)
$$

for all $x, y \in M_{k}$, and $0 \leq t \leq T$, where $r_{k}$ is the distance function of $M_{k}$.

Let $r(x, y)$ be the distance function of $M$, then by the choices of $\Omega_{k}$ and the construction of $M_{k}$, we have $r(x, y)=r_{k}(x, y)$ for all $x, y \in \Omega_{k-1}$.

Let $h \in C^{\infty}(M, N)$. By [H], for each $k$, there exists a unique solution $f_{k}$ of

$$
\begin{cases}\Delta u^{\alpha}-\frac{\partial u^{\alpha}}{\partial t}=-g^{i j} & \frac{\partial u^{\beta}}{\partial x^{i}} \frac{\partial u^{\gamma}}{\partial x^{j}} \Gamma_{\beta \gamma}^{\prime \alpha} \\ & \text { in } \Omega_{k} \times(0, \infty), \alpha=1, \ldots, n ; \\ u(x, 0)=h(x) & \text { in } \Omega_{k} ; \text { and } \\ u(x, t)=h(x) & \text { on } \partial \Omega_{k} \times[0, \infty) .\end{cases}
$$

LEMMA 1.3. For any $T>0$, and for any compact set $K \subset \subset \Omega \subset$ $M$, there exists a constant $C>0$ and an integer $k_{0}>0$ such that if $k \geq k_{0}$, then $e\left(f_{k}\right)(x, t) \leq C\left(E(h)+\sup _{\Omega} e(h)\right)$ for all $(x, t)$ $\in K \times[0, T]$ and $e\left(f_{k}\right)(x, t) \leq C E(h)$ for all $x \in K, 2 T \geq t \geq T$.

Proof. Obviously, it is sufficient to consider $K$ which is of the form $B_{x}\left(\frac{R}{2}\right)$ such that $R$ is less than the injectivity radius of $x$, where $B_{x}(r)$ is the geodesic ball of radius $r$ with center at $x$. Choose $k_{0}$ large enough so that $\overline{B_{x}(R)} \subset \Omega_{k}$ for all $k \geq k_{0}-1$. By the computation in [E-S], using the fact that $N$ has non-positive curvature, there exists a constant $C_{1}$ independent of $k$ such that for $k \geq k_{0}$

$$
\Delta_{M} e\left(f_{k}\right)-\frac{\partial}{\partial t} e\left(f_{k}\right) \geq-C_{1} e\left(f_{k}\right)
$$

on $B_{x}(R) \times[0, \infty)$.

Let $g_{k}=e\left(f_{k}\right) \exp \left(-C_{1} t\right)$. Then $g_{k}$ satisfies for $k \geq k_{0}$ :

$$
\Delta g_{k}-\frac{\partial g_{k}}{\partial t} \geq 0 \quad \text { on } B_{x}(R) \times[0, \infty)
$$

Since $R<$ injectivity radius of $x$, so we can find a smooth function $\eta: M \rightarrow[0,1]$ such that $\eta \equiv 1$ on $B_{x}\left(\frac{R}{2}\right), \eta \equiv 0$ outside $B_{x}(R)$. Hence $\eta(y) g_{k}(y, t)$ is smooth on $M \times[0, \infty)$. 
Furthermore, the support of $\eta(\cdot) g_{k}(\cdot, t)$ is contained in $\overline{B_{x}(R)}$ for all $t$. Hence $\eta(y) g_{k}(y, t)$ can be considered as a function on $M_{k_{0}} \times[0, \infty)$. By the uniqueness theorem of Cauchy problem in [K-L], noting that the volume of $M_{k_{0}}$ grows linearly, we have for $y \in B_{x}\left(\frac{R}{2}\right), 0 \leq t<\infty$,

$$
\begin{aligned}
g_{k}(y, t)= & \eta(y) g_{k}(y, t) \\
= & -\int_{0}^{t} d \tau \int_{M_{k_{0}}} H_{k_{0}}(y, z, t-\tau) \\
& \times\left(\Delta_{k_{0}}-\frac{\partial}{\partial \tau}\right)\left(\eta(z) g_{k}(z, \tau)\right) d V_{M_{k_{0}}}(z) \\
& +\int_{M_{k_{0}}} H_{k_{0}}(y, z, t) \eta(z) g_{k}(z, 0) d V_{M_{k_{0}}}(z),
\end{aligned}
$$

where $\Delta_{k_{0}}$ is the Laplace-Beltrami operator of $M_{k_{0}}$. Since the support of $\eta(y) g_{k}(y, t)$ is contained in $\overline{B_{x}(R)} \subset \Omega_{k_{0}-1}$, it is easy to see by (1.5) that

$$
\begin{aligned}
\left(\Delta_{k_{0}}\right. & \left.-\frac{\partial}{\partial \tau}\right)\left(\eta(z) g_{k}(z, \tau)\right)=\left(\Delta_{M}-\frac{\partial}{\partial \tau}\right)\left(\eta(z) g_{k}(z, \tau)\right) \\
= & \eta(z)\left(\Delta_{M}-\frac{\partial}{\partial \tau}\right) g_{k}(z, \tau)+\left(\Delta_{M} \eta(z)\right) g_{k}(z, \tau) \\
& +2\left\langle\nabla \eta(z), \nabla g_{k}(z, \tau)\right\rangle \\
\geq & \left(\Delta_{M} \eta(z)\right) g_{k}(z, \tau)+2\left\langle\nabla \eta(z), \nabla g_{k}(z, \tau)\right\rangle .
\end{aligned}
$$

Hence

$$
\begin{aligned}
g_{k}(y, t) \leq & -\int_{0}^{t} d \tau \int_{B_{x}(R)} H_{k_{0}}(y, z, t-\tau) \\
& \times\left(\Delta_{M} \eta(z)\right) g_{k}(z, \tau) d V_{M}(z) \\
& -2 \int_{0}^{t} d \tau \int_{B_{x}(R)} H_{k_{0}}(y, z, t-\tau) \\
& \times\left\langle\nabla \eta(z), \nabla g_{k}(z, \tau)\right\rangle d V_{M}(z) \\
& +\int_{B_{x}(R)} H_{k_{0}}(y, z, t) \eta(z) g_{k}(z, 0) d V_{M}(z) \\
= & \mathrm{I}+\mathrm{II}+\mathrm{III}
\end{aligned}
$$

where we have used the fact that in $\Omega_{k_{0}}$, the matrices of $M$ and $M_{k_{0}}$ are the same. Using the same fact, Lemma 1.2 and the fact that $\Delta_{M} \eta \equiv 0$ on $B_{x}\left(\frac{R}{2}\right)$, for any $T>0$, there exist $C_{2}, C_{3}$ and $C_{4}>0$ 
such that if $k \geq k_{0}$ and $0 \leq t \leq T$, then

$$
\begin{aligned}
\mathrm{I} & \leq C_{2} \int_{0}^{t} d \tau \int_{B_{x}(R)-B_{x}\left(\frac{R}{2}\right)}(t-\tau)^{-\frac{m}{2}} \exp \left(-\frac{C_{3} R^{2}}{4(t-\tau)}\right) g_{k}(z, \tau) d V_{M}(z) \\
& \leq C_{4} \sup _{0 \leq \tau \leq T} \int_{B_{x}(R)-B_{x}\left(\frac{R}{2}\right)} g_{k}(z, \tau) d V_{M}(z) .
\end{aligned}
$$

By $\left[\mathbf{H}\right.$, p. 135], the definition of $g_{k}$, we have

$$
\begin{aligned}
\mathrm{I} & \leq C_{4} \sup _{0 \leq \tau \leq T} \int_{B_{x}(R)-B_{x}\left(\frac{R}{2}\right)} e\left(f_{k}\right)(z, \tau) d V_{M}(z) \\
& \leq C_{4} \sup _{0 \leq \tau \leq T} \int_{\Omega_{k}} e\left(f_{k}\right)(z, \tau) d V_{M}(z) \\
& \leq C_{4} \int_{\Omega_{k}} e(h)(z) d V_{M}(z) \\
& \leq C_{4} E(h) .
\end{aligned}
$$

Similarly, integrating by parts in II and use the estimate for the gradient of $H_{k_{0}}(y, z, t)$ in Lemma 1.2 , for any $T>0$, we can find $C_{5}$ such that for $k \geq k_{0}$,

$$
\mathrm{II} \leq C_{5} E(h)
$$

Also

$$
\mathrm{III} \leq \sup _{B_{x}(R)} e(h) \int_{M_{k_{0}}} H_{k_{0}}(y, z, t) d V_{M_{k_{0}}}(z)=\sup _{B_{x}(R)} e(h),
$$

and

$$
\text { III } \leq C_{6} T^{-\frac{m}{2}} E(h) \quad \text { if } t \geq T .
$$

Combining (1.7)-(1.11), the lemma is proved.

Let us imbed $N$ isometrically in $\mathbf{R}^{q}$ for some $q$. This can be done because $N$ is compact. For $\Omega \subset M$, a map $u: \Omega \times[0, T) \rightarrow N \subset \mathbf{R}^{q}$ satisfies (1.1) in $\Omega \times[0, T)$ if and only if

$$
\left\{\begin{array}{c}
\Delta_{M} u^{A}-\frac{\partial u^{A}}{\partial t}=g^{i j} \mathbf{B}_{u(x, t)}\left(\frac{\partial u}{\partial x^{i}}, \frac{\partial u}{\partial x^{j}}\right), \\
\text { in } \Omega \times(0, T), A=1, \ldots, q ; \text { and } \\
u(x, 0)=h(x) \text { in } \Omega,
\end{array}\right.
$$

where $u=\left(u^{1}, \ldots, u^{q}\right)$ and $\mathbf{B}$ is the second fundamental form of $N$ in $\mathbf{R}^{q}$.

Before we state the next lemma, let us introduce the following notations. Let $\Omega$ be a domain in $\mathbf{R}^{m}$ and $T_{2}>T_{1} \geq 0, u=u(x, t)$ is a 
function defined on $Q_{T_{1}, T_{2}}=\Omega \times\left(T_{1}, T_{2}\right)$. For any positive number $l$, define

$$
|u|_{Q_{T_{1}, T_{2}}}^{(l)}=\langle u\rangle_{Q_{T_{1}, T_{2}}}^{(l)}+\sum_{j=1}^{[l]}\langle u\rangle_{Q_{T_{1}, T_{2}}}^{(j)},
$$

where $[l]=$ integral part of $l$, and

$$
\begin{aligned}
& \langle u\rangle_{Q_{T_{1}, T_{2}}^{(0)}}^{(0)} \equiv|u|_{Q_{T_{1}, T_{2}}}^{(0)}=\max _{Q_{T_{1}, T_{2}}}|u|, \\
& \langle u\rangle_{Q_{T_{1}, T_{2}}}^{(j)}=\sum_{2 r+s=j}\left|D_{t}^{r} D_{x}^{s} u\right|_{Q_{T_{1}, T_{2}}}^{(0)}, \\
& \langle u\rangle_{Q_{T_{1}, T_{2}}}^{(l)}=\langle u\rangle_{x, Q_{T_{1}, T_{2}}}^{(l)}+\langle u\rangle_{t, Q_{T_{1}, T_{2}}^{(l / 2)},}, \\
& \langle u\rangle_{x, Q_{T_{1}, T_{2}}}^{(l)}=\sum_{2 r+s=[l]}\left\langle D_{t}^{r} D_{x}^{s} u\right\rangle_{x, Q_{T_{1}, T_{2}}^{l-[l]}}^{l}, \\
& \langle u\rangle_{t, Q_{T_{1}, T_{2}}^{l / 2}}^{l / 2} \sum_{0<l-2 r-s<2}\left\langle D_{t}^{r} D_{x}^{s} u\right\rangle_{t, Q_{T_{1}, T_{2}}^{\left(\frac{l-2 r-s}{2}\right)}}, \\
& \langle u\rangle_{x, Q_{T_{1}, T_{2}}^{(\alpha)}}^{(\alpha)} \sup _{(x, t),\left(x^{\prime}, t\right) \in Q_{T_{1}, T_{2}}} \frac{\left|u(x, t)-u\left(x^{\prime}, t\right)\right|}{\left|x-x^{\prime}\right|^{\alpha}}, \quad 0<\alpha<1, \\
& \langle u\rangle_{t, Q_{T_{1}, T_{2}}}^{(\alpha)}=\sup _{(x, t),\left(x, t^{\prime}\right) \in Q_{T_{1}, T_{2}}} \frac{\left|u(x, t)-u\left(x, t^{\prime}\right)\right|}{\left|t-t^{\prime}\right|^{\alpha}}, \quad 0<\alpha<1 .
\end{aligned}
$$

LEMMA 1.4. Let the sequence of maps $f_{k}: \Omega_{k} \rightarrow N \subset \mathbf{R}^{q}$ as in Lemma 1.3. Write $f_{k}=\left(f_{k}^{1}, \ldots, f_{k}^{q}\right)$. Given any compact subdomain $K$ of a coordinate neighborhood of some point with coordinates $\left(x^{1}, \ldots, x^{m}\right)$, given $T_{2}>T_{1}>0$ and given any positive integer $l$, there exist constants $C>0,1>\alpha>0$ and positive integer $k_{0}$, such that if $k \geq k_{0}$ then

$$
\left|f_{k}^{A}\right|_{K_{T_{1}, T_{2}}}^{l+\alpha} \leq C
$$

for $A=1, \ldots, q$.

Proof. This follows from Lemma 1.3 , the fact that $f_{k}^{A}$ are uniformly bounded, the results of Hölder estimates of the gradients and Schauder estimates of the solutions of parabolic equations. See, for example [L-S-U, p. 210, Theorem 11.1 and p. 352, Theorem 10.1].

Proof of Theorem 1.1. Let $f_{k}$ be the sequence of maps as in Lemma 1.3. By Lemmas 1.3 and 1.4 , we can find a subsequence of $f_{k}$, which 
we also denote by $f_{k}$, such that $f_{k}$ together with their first and second derivatives with respect to the space variable, first derivative with respect to the time variable, converge uniformly on compact subsets of $M \times(0, \infty)$ to some $f$ and its derivatives. Obviously $f$ is a solution of the heat flow in $(1.1)$ on $M \times(0, \infty)$. In order to prove that $\lim _{t \rightarrow 0} f(x, t)=h(x)$, note that for any $x \in M$, and $T>0$, by [L-S-U, p. 204] and Lemma 1.3, there exists $1>\alpha>0, C>0$ and a positive integer $k_{0}$ such that for $k \geq k_{0}, 0<t<T$,

$$
\left|f_{k}^{A}(x, t)-f_{k}^{A}(x, 0)\right| \leq C t^{\alpha}, \quad A=1, \ldots, q,
$$

where as before $N$ is embedded in $\mathbf{R}^{q}$. Since $f_{k}^{A}(x, 0)=h^{A}(x)$ is the initial data, therefore if we let $k \rightarrow \infty$ we have

$$
\left|f^{A}(x, t)-h^{A}(x)\right| \leq C t^{\alpha}, \quad A=1, \ldots, q, 0 \leq t \leq T .
$$

Hence $f$ is in fact a solution of (1.1). The proof of Theorem 1.1 is then completed.

2. Properties of solutions of (1.1). Let us first prove a uniqueness theorem for the solutions of (1.1). We need a maximum principle which is a variant of a theorem in [K-L].

LEMMA 2.1. Let $M$ be a complete noncompact Riemannian manifold such that there exists a point $p \in M$ and a constant $k>0$ satisfying

$$
\operatorname{Vol}\left(B_{p}(r)\right) \leq \exp \left(k\left(1+r^{2}\right)\right)
$$

for all $r>0$. Let $f$ be a function on $M \times[0, T), T>0$. $f$ is smooth on $M \times(0, T)$ and continuous on $M \times[0, T)$. Suppose $f$ satisfies the following conditions:

(a) $\left(\Delta-\frac{\partial}{\partial t}\right) f \geq 0$ on $M \times(0, T)$;

(b) $f(x, 0) \leq 0$ for all $x \in M$; and

(c) $\int_{0}^{T}\left(\int_{M} \exp \left(-\alpha r^{2}(p, y)\right)|\nabla f|^{2}(y) d V_{M}(y)\right) d t<\infty$, for some $\alpha>0$.

Then $f \leq 0$ on $M \times[0, T)$.

Proof. Let $0<\eta<\min \left(T, \frac{1}{8 \alpha}, \frac{1}{16 k}\right)$ be a fixed constant. Define

$$
g(y, s)=-\frac{r^{2}(p, y)}{4(2 \eta-s)},
$$

where $r(p, y)$ is the distance between $p$ and $y$, and $0<s<\eta$. It is easy to check

$$
|\nabla g|^{2}+\frac{\partial g}{\partial s} \equiv 0 \quad \text { on } M \times(0, \eta)
$$


For $K>0$, let $f_{K}=\max \{\min (f, K), 0\}$. Hence

$$
f_{K}(x, t)= \begin{cases}K, & \text { if } f(x, t) \geq K, \\ f(x, t), & \text { if } 0<f(x, t)<K, \\ 0, & \text { if } f(x, t) \leq 0 .\end{cases}
$$

$f_{K}$ is uniformly Lipschitz on any compact subset of $M \times(0, T)$. For $0<t<T$, let

$$
M_{t}=\{x \in M \mid f(x, t)>0\} .
$$

For any smooth function $\varphi$ on $M$ with compact support, by assumption (a), for $0<\varepsilon<\eta$,

$$
\int_{\varepsilon}^{\eta}\left(\int_{M} \varphi^{2} e^{g} f_{K}\left(\Delta f-\frac{\partial f}{\partial s}\right) d V_{M}\right) d s \geq 0,
$$

where we have used the fact that $f_{K} \geq 0$. Hence

$$
\begin{aligned}
0 \leq & -\int_{\varepsilon}^{\eta}\left(\int_{M} \varphi^{2} e^{g}\left\langle\nabla f_{K}, \nabla f\right\rangle d V_{M}\right) d s \\
& -\int_{\varepsilon}^{\eta}\left(\int_{M} \varphi^{2} e^{g} f_{K}\langle\nabla g, \nabla f\rangle d V_{M}\right) d s \\
& -2 \int_{\varepsilon}^{\eta}\left(\int_{M} \varphi e^{g} f_{K}\langle\nabla \varphi, \nabla f\rangle d V_{M}\right) d s \\
& -\int_{\varepsilon}^{\eta}\left(\int_{M} \varphi^{2} e^{g} f_{K} \frac{\partial f}{\partial s} d V_{M}\right) d s \\
= & \mathrm{I}+\mathrm{II}+\mathrm{III}+\mathrm{IV} .
\end{aligned}
$$

$$
\mathrm{I}=-\int_{\varepsilon}^{\eta}\left(\int_{M_{s}} \varphi^{2} e^{g}\left|\nabla f_{K}\right|^{2} d V_{M}\right) d s
$$

$$
\begin{aligned}
\mathrm{II} \leq & \frac{1}{2} \int_{\varepsilon}^{\eta}\left(\int_{M} \varphi^{2} e^{g}|\nabla f|^{2} d V_{M}\right) d s \\
& +\frac{1}{2} \int_{\varepsilon}^{\eta}\left(\int_{M} \varphi^{2} e^{g}|\nabla g|^{2} f_{K}^{2} d V_{M}\right) d s .
\end{aligned}
$$

$$
\begin{aligned}
\mathrm{III} \leq & \frac{1}{2} \int_{\varepsilon}^{\eta}\left(\int_{M_{s}} \varphi^{2} e^{g}|\nabla f|^{2} d V_{M}\right) d s \\
& +2 \int_{\varepsilon}^{\eta}\left(\int_{M} e^{g} f_{K}^{2}|\nabla \varphi|^{2} d V_{M}\right) d s .
\end{aligned}
$$


To estimate IV, note that

(2.7) $e^{g} f_{K}\left(\frac{\partial f_{K}}{\partial s}-\frac{\partial f}{\partial s}\right)=\frac{\partial}{\partial s}\left\{e^{g} f_{K}\left(f_{K}-f\right)\right\}$

$$
-e^{g} \frac{\partial g}{\partial s} f_{K}\left(f_{K}-f\right)-e^{g} \frac{\partial f_{K}}{\partial s}\left(f_{K}-f\right) .
$$

From (2.2), we see that

$$
\frac{\partial f_{K}}{\partial s}\left(f_{K}-f\right)=0
$$

whenever $\frac{\partial f_{K}}{\partial s}$ exists.

By (2.1) and (2.2) we also have

$$
\frac{\partial g}{\partial s} f_{K}\left(f_{K}-f\right) \geq 0
$$

Hence (2.7) gives

$$
-e^{g} f_{K} \frac{\partial f}{\partial s} \leq-e^{g} f_{K} \frac{\partial f_{K}}{\partial s}+\frac{\partial}{\partial s}\left\{e^{g} f_{K}\left(f_{K}-f\right)\right\},
$$

whenever $\frac{\partial f_{K}}{\partial s}$ exists.

Since $f_{K}$ is uniformly Lipschitz on compact subsets of $M \times(0, T)$, therefore

$$
\begin{aligned}
\mathrm{IV} \leq & -\int_{\varepsilon}^{\eta}\left(\int_{M} \varphi^{2} e^{g} f_{K} \frac{\partial f_{K}}{\partial s} d V_{M}\right) d s \\
& +\int_{\varepsilon}^{\eta}\left(\int_{M} \varphi^{2} \frac{\partial}{\partial s}\left\{e^{g} f_{K}\left(f_{K}-f\right)\right\} d V_{M}\right) d s \\
= & -\left.\frac{1}{2} \int_{M} \varphi^{2} e^{g} f_{K}^{2} d V_{M}\right|_{s=\eta}+\left.\frac{1}{2} \int_{M} \varphi^{2} e^{g} f_{K}^{2} d V_{M}\right|_{s=\varepsilon} \\
& +\frac{1}{2} \int_{\varepsilon}^{\eta}\left(\int_{M} \varphi^{2} e^{g} f_{K}^{2} \frac{\partial g}{\partial s} d V_{M}\right) d s \\
& +\left.\int_{M} \varphi^{2} e^{g} f_{K}\left(f_{K}-f\right) d V_{M}\right|_{s=\eta} \\
& -\left.\int_{M} \varphi^{2} e^{g} f_{K}\left(f_{K}-f\right) d V_{M}\right|_{s=\varphi} .
\end{aligned}
$$

Combining (2.1), (2.3), (2.4), (2.5), (2.6) and (2.9), and letting 
$\varepsilon \rightarrow 0$, we have

$$
\begin{aligned}
0 \leq & -\int_{0}^{\eta}\left(\int_{M_{s}} \varphi^{2} e^{g}\left|\nabla f_{K}\right|^{2} d V_{M}\right) d s \\
& +\int_{0}^{\eta}\left(\int_{M_{s}} \varphi^{2} e^{g}|\nabla f|^{2} d V_{M}\right) d s \\
& +2 \int_{0}^{\eta}\left(\int_{M} e^{g} f_{K}^{2}|\nabla \varphi|^{2} d V_{M}\right) d s-\left.\frac{1}{2} \int_{M} \varphi^{2} e^{g} f_{K}^{2} d V_{M}\right|_{s=\eta},
\end{aligned}
$$

where we have used the fact that $f_{K}\left(f_{K}-f\right) \leq 0$ and that $f_{K} \equiv 0$ at $s=0$.

Hence

(2.10)

$$
\begin{aligned}
\frac{1}{2} \int_{M} & \left.\varphi^{2} e^{g} f_{K}^{2} d V_{M}\right|_{s=\eta} \\
\leq & \int_{0}^{\eta}\left(\int_{M_{s}} \varphi^{2} e^{g}\left(|\nabla f|^{2}-\left|\nabla f_{K}\right|^{2}\right) d V_{M}\right) d s \\
& +2 \int_{0}^{\eta}\left(\int_{M} e^{g} f_{K}^{2}|\nabla \varphi|^{2} d V_{M}\right) d s .
\end{aligned}
$$

For $R>0$, let $\varphi$ be such that $0 \leq \varphi \leq 1 ; \varphi \equiv 1$ on $B_{p}(R) ; \varphi \equiv 0$ outside $B_{p}(R+1)$ and $|\nabla \varphi| \leq 2$, we have

$$
\begin{aligned}
\left.\frac{1}{2} \int_{B_{p}(R)} e^{g} f_{K}^{2} d V_{M}\right|_{s=\eta} \\
\leq \int_{0}^{\eta}\left(\int_{B_{p}(R+1) \cap M_{s}} e^{g}\left(|\nabla f|^{2}-\left|\nabla f_{K}\right|^{2}\right) d V_{M}\right) d s \\
+8 \int_{0}^{\eta}\left(\int_{B_{p}(R+1)-B_{p}(R)} e^{g} f_{K}^{2} d V_{M}\right) d s .
\end{aligned}
$$

Since $0<\eta<\min \left(\frac{1}{16 k}, \frac{1}{8 \alpha}\right)$, so $g(y, s) \leq-2 k r^{2}(p, y)$ and $g(y, s)$ $\leq-\alpha r^{2}(p, y)$ for all $0<s<\eta$. Also $f_{K}^{2} \leq K^{2}$. By the assumption on the volume growth of $M$, it is easy to see that the second term on the right side of (2.11) tends to zero as $R \rightarrow \infty$. Since $0 \leq$ $|\nabla f|^{2}-\left|\nabla f_{K}\right|^{2} \leq|\nabla f|^{2}$, by assumption (c) if we let $R \rightarrow \infty$ in (2.11), we obtain

$$
\begin{aligned}
& \left.\frac{1}{2} \int_{M} e^{g} f_{K}^{2} d V_{M}(y)\right|_{s=\eta} \\
& \quad \leq \int_{0}^{\eta}\left(\int_{M_{s}} e^{g}\left(|\nabla f|^{2}-\left|\nabla f_{K}\right|^{2}\right) d V_{M}(y)\right) d s
\end{aligned}
$$


Since $f_{K}^{2}$ approaches $\left(f^{+}\right)^{2}$ as $K \rightarrow \infty$, where $f^{+}=\max (f, 0)$, and for all $s,\left|\nabla f_{K}\right|^{2} \rightarrow|\nabla f|^{2}$ on $M_{s}$, by (c) again, if we let $K \rightarrow \infty$ in (2.12), we have

$$
\left.\frac{1}{2} \int_{M} e^{g}\left(f^{+}\right)^{2} d V_{M}(y)\right|_{s=\eta} \leq 0 .
$$

Hence $f^{+} \equiv 0$ at $t=\eta$. Since $\eta$ is any number satisfying $0<\eta<$ $\min \left(T, \frac{1}{8 \alpha}, \frac{1}{16 k}\right)$, it is easy to conclude inductively that $f^{+} \equiv 0$ on $M \times(0, T)$. Hence $f \leq 0$ on $M \times[0, T)$.

REMARK 2.2. $M$ will satisfy the volume growth condition in the lemma, if there exists a constant $C>0$ such that the Ricci curvature at every point $x \in M$ satisfies $\operatorname{Ric}(x) \geq-C\left(1+r^{2}(p, x)\right)$, see [K-L].

THEOREM 2.3. Let $M$ be a complete non-compact Riemannian manifold satisfying the volume growth condition in Lemma 2.1. Let $N$ be a complete Riemannian manifold with non-positive curvature. Suppose $u_{1}$ and $u_{2}$ are two maps from $M \times[0, T)$ to $N$ satisfying (1.1) with the same initial condition. Suppose there exists a point $p \in M$ and $\alpha>0$ such that

$$
\int_{0}^{T}\left(\int_{M} \exp \left(-\alpha r^{2}\right) e\left(u_{i}\right) d V_{M}\right) d s<\infty
$$

for $i=1,2$, where $r=r(p, y)$. Then $u_{1} \equiv u_{2}$ on $M \times[0, T)$.

Proof. For any $0<t<T$ and any $x \in M$, let $\gamma$ be the geodesic joining $u_{1}(x, t)$ and $u_{2}(x, t)$ which is homotopic to $f:[0,2 t] \rightarrow N$, where

$$
f(\tau)= \begin{cases}u_{1}(x, t-\tau), & 0 \leq \tau \leq t, \\ u_{2}(x, \tau-t), & t<\tau \leq 2 t .\end{cases}
$$

Since $N$ is non-positively curved, $\gamma$ is unique. Let $\rho(x, t)$ be the length of $\gamma$, then $\rho^{2}$ is smooth on $M \times(0, T)$. We should remark tht the function $\rho$ may not be bounded even if $N$ is compact.

Let $\psi=\left(\rho^{2}+1\right)^{1 / 2}-1 \geq 0$, then by [S-Y2, p. 369],

$$
|\nabla \psi|^{2} \leq 2\left(e\left(u_{1}\right)+e\left(u_{2}\right)\right) \text {. }
$$

Hence

$$
\int_{0}^{T}\left(\int_{M} \exp \left(-\alpha r^{2}\right)|\nabla \psi|^{2} d V_{M}\right) d t<\infty
$$

by the assumption on $u_{1}$ and $u_{2}$. Since $u_{1}$ and $u_{2}$ satisfy (1.1), as in [S-Y2, pp. 368-369], one can obtain

$$
\left(\Delta_{M}-\frac{\partial}{\partial t}\right) \psi \geq 0 \text { on } M \times(0, T) .
$$


Note that $\psi(x, 0) \equiv 0$ on $M$. Hence by Lemma 2.1 , we have $\psi \equiv 0$ on $M \times[0, T)$. That is, $\rho \equiv 0$, and $u_{1} \equiv u_{2}$ on $M \times[0, T)$.

Next we study the monotonicty of total energy. Let $M^{m}$ and $N^{n}$ be complete Riemannian mainifolds, $M$ is non-compact. Let $u: M \times(0, T) \rightarrow N$ be a smooth map satisfying the heat equation (1.1) for harmonic maps. Let $p \in M$ be a fixed point. For $0<t<T$ and $R>0$, let $E(t, R)=\int_{B_{p}(R)} e(u(\cdot, t)) d V_{M}$, and $\bar{E}(t, R)=\sup _{0<\tau<t} E(\tau, R)$. Also $E(t)=\int_{M} e(u(\cdot, t)) d V_{M}$.

THEOREM 2.4. Suppose there exists a constant $k>0$ such that $\bar{E}(T, R) \leq \exp (k(1+R))$ for all $R>0$. Then $E(t)$ is a non-increasing function in $t$. More precisely, for $0<t_{1}<t_{2}<T$, if $E\left(t_{1}\right)<\infty$, then

$$
E\left(t_{2}\right)+2 \int_{t_{1}}^{t_{2}} d t \int_{M}\left|u_{t}\right|^{2} d_{M} \leq E\left(t_{1}\right)<\infty .
$$

REMARK 2.5. The condition of the theorem will be satisfied if (1) $\sup _{0<t<T} E(t)<\infty$ or (2) $M$ has at most exponential volume growth and $\sup _{0<t<T ; x \in B_{p}(R)} e(u(x, t))$ is less than or equal to $\exp (C(1+R))$ for some $C>0$. Note tht if the Ricci curvature of $M$ is bounded below by $-K$, then $M$ has at most exponential volume growth.

Proof of Theorem 2.4. It is more convienent to use moving frame. Let $f: M \rightarrow N$ be a smooth map, and let $\theta_{1}, \ldots, \theta_{m}$ be an orthonormal coframe in a neighborhood of some point $q \in M$. Let $\omega_{1}, \ldots, \omega_{n}$ be an orthonormal coframe in a neighborhood of $f(q)$. We have the structure equations for $M$ and $N$

$$
d \theta_{i}=\sum_{j} \theta_{i j} \wedge \theta_{j}, \quad 1 \leq i \leq m
$$

and

$$
d \omega_{\alpha}=\sum_{\beta} \omega_{\alpha \beta} \wedge \omega_{\beta}, \quad 1 \leq \alpha \leq n .
$$

Define $f_{i}^{\alpha}$ and $f_{i j}^{\alpha}, \quad 1 \leq i, j \leq m, 1 \leq \alpha \leq n$, by

$$
\begin{gathered}
f^{*}\left(\omega_{\alpha}\right)=\sum_{i} f_{i}^{\alpha} \theta_{i}, \\
d f_{i}^{\alpha}+\sum_{\beta} f_{i}^{\beta} f^{*}\left(\omega_{\beta \alpha}\right)+\sum_{j} f_{i}^{\alpha} \theta_{j i}=\sum_{j} f_{i j}^{\alpha} \theta_{j} .
\end{gathered}
$$


In our case $u(x, t)$ is a map from $M \times(0, T)$ to $N$. Let $d t$ be the unit covector in the $t$ direction, then $u_{t}^{\alpha}$ is defined by

$$
u^{*}\left(\omega_{\alpha}\right)=\sum_{i} u_{i}^{\alpha} \theta_{i}+u_{t}^{\alpha} d t, \quad 1 \leq \alpha \leq n .
$$

Then

$$
e(u(\cdot, t))=\sum_{i, \alpha}\left(u_{i}^{\alpha}\right)^{2}(\cdot, t), \quad 1 \leq i \leq m, 1 \leq \alpha \leq n .
$$

Also we have

$$
\sum_{i} u_{i i}^{\alpha}-u_{t}^{\alpha}=0, \quad 1 \leq \alpha \leq n
$$

Let $\varphi$ be a smooth funciton on $M$ with compact support. By (2.13) and (2.14), for any $\varepsilon>0$,

$$
\begin{aligned}
\frac{d}{d t} \int_{M} e(u(\cdot, t)) \varphi^{2} d V_{M} \\
=2 \int_{M}\left(\sum_{i, \alpha} u_{i}^{\alpha} u_{i t}^{\alpha}\right) \varphi^{2} d V_{M}=2 \int_{M}\left(\sum_{i, \alpha} u_{i}^{\alpha} u_{t i}^{\alpha}\right) \varphi^{2} d V_{M} \\
=-2 \int_{M}\left(\sum_{i, \alpha} u_{i i}^{\alpha} u_{t}^{\alpha}\right) \varphi^{2} d V_{M}-4 \int_{M} \sum_{i, \alpha}\left(u_{i}^{\alpha} u_{t}^{\alpha} \varphi_{i} \varphi\right) d V_{M} \\
\leq-2 \int_{M}\left(\sum_{\alpha}\left(u_{t}^{\alpha}\right)^{2}\right) \varphi^{2} d V_{M}+\varepsilon \int_{M}\left(\sum_{\alpha}\left(u_{t}^{\alpha}\right)^{2}\right) \varphi^{2} d V_{M} \\
\quad+C \int_{M} e(u(\cdot, t))|\nabla \varphi|^{2} d V_{M} \\
=C \int_{M} e(u(\cdot, t))|\nabla \varphi|^{2} d V_{M} \\
\quad+(-2+\varepsilon) \int_{M}\left(\sum_{\alpha}\left(u_{t}^{\alpha}\right)^{2}\right) \varphi^{2} d V_{M}
\end{aligned}
$$

where $C$ is a constant depending only on $m$ and $\varepsilon$. Without loss of generality, we may assume $u$ is smooth on $M \times[0, T)$ and show that $E(t)+2 \int_{0}^{t} d \tau \int_{M} \sum_{\alpha}\left(u_{\tau}^{\alpha}\right)^{2} d V_{M} \leq E(0)$ for all $T>t>0$. Hence, let 
us assume $E(0)<\infty$. Integrate $(2.15)$ from 0 to $t$,

$$
\begin{aligned}
\int_{M} e(u(\cdot, t)) \varphi^{2} d V_{M}-\int_{M} e(u(\cdot, 0)) \varphi^{2} d V_{M} \\
\leq C \int_{0}^{t} d \tau \int_{M} e(u(\cdot, \tau))|\nabla \varphi|^{2} d V_{M} \\
\quad+(-2+\varepsilon) \int_{0}^{t} d \tau \int_{M} \sum_{\alpha}\left(u_{\tau}^{\alpha}\right)^{2} \varphi^{2} d V_{M}
\end{aligned}
$$

For $R>0$, and for any positive integer $j$, let $\varphi$ be such that $0 \leq \varphi \leq 1, \varphi \equiv 1$ on $B_{p}(j R), \varphi \equiv 0$ outside $B_{p}((j+1) R)$ and $|\nabla \varphi| \leq \frac{2}{R}$. By $(2.16)$ we have

$$
\begin{aligned}
E(t, & j R)+(2-\varepsilon) \int_{0}^{t} d \tau \int_{B_{p}(j R)}\left(\sum_{\alpha}\left(u_{\tau}^{\alpha}\right)^{2}\right) d V_{M} \\
& \leq E(0,(j+1) R)+\frac{4 C}{R^{2}} \int_{0}^{t} E(\tau,(j+1) R) d \tau \\
& \leq E(0,(j+1) R)+\frac{4 C}{R^{2}} \int_{0}^{t} E(\tau,(j+1) R) d \tau
\end{aligned}
$$

We claim that for any integer $\nu>0$,

$$
\begin{aligned}
E(t, R) \leq & E(0,(\nu+1) R) \sum_{j=1}^{\nu} \frac{1}{j !}\left(\frac{4 C t}{R^{2}}\right)^{j-1} \\
& +\left(\frac{4 C}{R^{2}}\right)^{\nu} \int_{0}^{t} d s_{1} \int_{0}^{s_{1}} d s_{2} \cdots \\
& \int_{0}^{s_{\nu-1}} E\left(s_{\nu},(\nu+1) R\right) d s_{\nu}
\end{aligned}
$$

By $(2.17),(2.18)$ is obviously true for $\nu=1$. Suppose $(2.18)$ is true for $\nu$. By (2.17)

$$
\begin{aligned}
E\left(s_{\nu},(\nu+1) R\right) \leq & E(0,(\nu+2) R) \\
& +\frac{4 C}{R^{2}} \int_{0}^{s_{\nu}} E\left(s_{\nu+1},(\nu+2) R\right) d s_{\nu+1} .
\end{aligned}
$$


Hence

$$
\begin{aligned}
E(t, R) \leq & E(0,(\nu+1) R) \sum_{j=1}^{\nu} \frac{1}{j !}\left(\frac{4 C t}{R^{2}}\right)^{j-1} \\
& +\left(\frac{4 C}{R^{2}}\right)^{\nu} \int_{0}^{t} d s_{1} \int_{0}^{s_{1}} d s_{2} \ldots \\
& \int_{0}^{s_{\nu-1}}\{E(0,(\nu+2) R) \\
& \left.+\frac{4 C}{R^{2}} \int_{0}^{s_{\nu}} E\left(s_{\nu+1},(\nu+2) R\right) d s_{\nu+1}\right\} d s_{\nu} \\
\leq & E(0,(\nu+2) R) \sum_{j=1}^{\nu+1} \frac{1}{j !}\left(\frac{4 C t}{R^{2}}\right)^{j-1} \\
& +\left(\frac{4 C}{R^{2}}\right)^{\nu+1} \int_{0}^{t} d s_{1} \cdots \int_{0}^{s_{\nu}} E\left(s_{\nu+1},(\nu+2) R\right) d s_{\nu+1} .
\end{aligned}
$$

Hence (2.18) is true for all $\nu$. Replace $R$ by $2 R$ in (2.18) and let $j=1$ in $(2.17)$, we have

$$
\begin{aligned}
E(2.19) & R)+(2-\varepsilon) \int_{0}^{t} d \tau \int_{B_{p}(R)}\left(\sum_{\alpha}\left(u_{\tau}^{\alpha}\right)^{2}\right) d V_{M} \\
\leq & E(0,2(\nu+1) R) \sum_{j=1}^{\nu} \frac{1}{j !}\left(\frac{C t}{R^{2}}\right)^{j-1} \\
& +\bar{E}(t, 2(\nu+1) R)\left(\frac{C}{R^{2}}\right)^{\nu} \int_{0}^{t} d s_{1} \cdots \int_{0}^{s_{\nu-1}} d s_{\nu} \\
\leq & E(0) \sum_{j=1}^{\nu} \frac{1}{j !}\left(\frac{C t}{R^{2}}\right)^{j-1}+\bar{E}(t, 2(\nu+1) R)\left(\frac{C}{R^{2}}\right)^{\nu} \cdot \frac{t^{\nu}}{\nu !} \\
\leq & E(0) \exp \left(\frac{C t}{R^{2}}\right)+\exp (k(1+2(\nu+1) R)) \cdot\left(\frac{C t}{R^{2}}\right)^{\nu} \cdot \frac{1}{\nu !} .
\end{aligned}
$$

For $0<t<T$ and $R$ fixed, if we let $\nu \rightarrow \infty$ in (2.19), by the Stirling's formula $\nu ! \sim \sqrt{2 \pi} \nu^{\nu+\frac{1}{2}} e^{-\nu}$ as $\nu \rightarrow \infty$, we conclude that

$$
E(t, R)+(2-\varepsilon) \int_{0}^{t} d \tau \int_{B_{p}(R)}\left(\sum_{\alpha}\left(u_{\tau}^{\alpha}\right)^{2}\right) d V_{M} \leq E(0) \cdot \exp \left(\frac{C t}{R^{2}}\right) .
$$

Let $R \rightarrow \infty$, and then let $\varepsilon \rightarrow \infty$, the theorem is then proved.

COROLlary 2.6. With the same assumptions as in Theorem 1.1 , let $u$ be the solution constructed in the theorem. Then $E(u(\cdot, t)) \leq E(h)$ for all $t>0$, and $E(u(\cdot, t))$ is non-increasing in $t$. 
Proof. By the construction of $u$ and the result in [H, p. 135], we have $E(u(\cdot, t)) \leq E(h)<\infty$ for all $t>0$. By Theorem 2.4, we can conclude that $E(u(\cdot, t))$ is non-increasing in $t$.

THEOREM 2.7. With the same assumptions as in Theorem 1.1 and letting $u$ be the solution of (1.1) obtained in Theorem 1.1. There exists $t_{j} \rightarrow \infty$ with $t_{j+1}>t_{j}+2$ such that $u\left(\cdot, t_{j}\right): M \rightarrow N$ converge together with their first and second derivatives in the space variable uniformly on compact subsets of $M$ to a harmonic map $u_{\infty}$.

Proof. Using Corollary 2.6, as in Lemma 1.3 one can prove that for any $R>0$ there exists a constant $C$ which is independent of $t$ such that $e(u)(x, t) \leq C$ for all $x \in B_{p}(R)$ and for all $t$. As in Lemma 1.4 , one can show that there exists $t_{j} \rightarrow \infty$ such that the sequence of maps $v_{j}(x, t)=u\left(x, t_{j}+t\right)$ from $M \times[0,1]$ to $N$ converge together with their first and second derivatives in the space variable and the first derivative of the time variable uniformly on $B_{p}(R) \times[0,1]$ for any $R>0$. By Theorem 2.4 and the fact that $u$ is a solution of (1.1), the result follows.

REMARK 2.8. $u_{\infty}$ in the above theorem is homotopic to $h$ on compact subsets.

Acknowledgment. The authors would like to thank Peter Li for many useful conversations.

\section{REFERENCES}

[C-L-Y] S. Y. Cheng, P. Li and S. -T. Yau, On the upper estimates of the heat kernel of a complete Riemannian manifold, Amer. J. Math., 103 (1981), 1021-1063.

[E-S] J. Eells and J. Sampson, Harmonic mappings of Riemannian manifolds, Amer. J. Math., 86 (1964), 109-160.

[H] R. Hamilton, Harmonic Maps of Manifolds with Boundary, Lecture Notes 471, Springer, 1975.

[Ht] P. Hartman, On homotopic harmonic maps, Canad. J. Math., 19 (1967), 673-687.

[K-L] K. Karp and P. Li, The heat equation on complete Riemannian manifolds, preprint.

[L-S-U] O. A. Ladyzenskaja, V. A. Solonnikov and N. N. Ural'ceva, Linear and quasilinear equations of parabolic type, Amer. Math. Soc. Transl. Math. Monographs 23, Providence, 1968.

[S-Y1] R. Schoen and S. -T. Yau, Harmonic maps and the topology of stable hypersurfaces and mamifolds with non-negative Ricci curvature, Comment. Math. Helvetici, 39 (1976), 333-341. 
[S-Y2] R. Schoen and S. -T. Yau, Compact group actions and the topology of manifolds with nonpositive curvature, Topology, 18 (1979), 361-380.

[Tr] A. Treibergs, Lecture on Eells-Sampson theorem on parabolic deformation of maps to harmonic maps, Univ. of Utah Notes (1987).

Received February 7, 1989 and in revised form March 19, 1991. Research by second author partially supported by the Earmarked Grant for Research, Hong Kong.

UNIVERSITY OF TEXAS

ARLINGTON, TX 76019

AND

UNIVERSITY OF CALIFORNIA

IRVINE, CA 92717 



\section{PACIFIC JOURNAL OF MATHEMATICS EDITORS}

V. S. VARADARAJAN

(Managing Editor)

University of California

Los Angeles, CA 90024-1555-05

Herbert Clemens

University of Utah

Salt Lake City, UT 84112

F. Michael Christ

University of California

Los Angeles, CA 90024-1555

THOMAS ENRIGHT

University of California, San Diego

La Jolla, CA 92093

\section{C. MOORE}

University of California

Berkeley, CA 94720

MARTIN SCHARLEMANN

University of California

Santa Barbara, CA 93106

HAROLD STARK

University of California, San Diego

La Jolla, CA 92093

STEVEN KERCKHOFF

Stanford University

Stanford, CA 94305

ASSOCIATE EDITORS

R. ARENS

E. F. BECKENBACH (1906-1982)

B. H. NeumanN

F. WOLF

(1904-1989)

K. YosHIDA

SUPPORTING INSTITUTIONS

UNIVERSITY OF ARIZONA

UNIVERSITY OF OREGON

UNIVERSITY OF BRITISH COLUMBIA

UNIVERSITY OF SOUTHERN CALIFORNIA

CALIFORNIA INSTITUTE OF TECHNOLOGY

UNIVERSITY OF CALIFORNIA

MONTANA STATE UNIVERSITY

STANFORD UNIVERSITY

UNIVERSITY OF NEVADA, RENO

UNIVERSITY OF HAWAII

NEW MEXICO STATE UNIVERSITY

OREGON STATE UNIVERSITY

UNIVERSITY OF UTAH

WASHINGTON STATE UNIVERSITY

UNIVERSITY OF WASHINGTON 


\section{Pacific Journal of Mathematics}

\section{Vol. 153, No. $1 \quad$ March, 1992}

Patrick Robert Ahern and Carmen Cascante, Exceptional sets for Poisson integrals of potentials on the unit sphere in $\mathbf{C}^{n}, p \leq 1 \ldots \ldots \ldots \ldots \ldots$

David Peter Blecher, The standard dual of an operator space ........... 15

Patrick Gilmer, Real algebraic curves and link cobordism .............. 31

Simon M. Goberstein, On orthodox semigroups determined by their

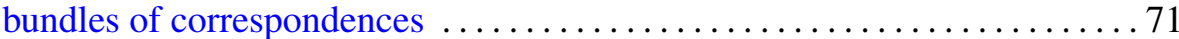

John Kalliongis and Darryl John McCullough, Homeotopy groups of irreducible 3-manifolds which may contain two-sided projective planes .......................................... 85

Yuji Konishi, Masaru Nagisa and Yasuo Watatani, Some remarks on actions of compact matrix quantum groups on $C^{*}$-algebras $\ldots \ldots \ldots \ldots 119$

Guojun Liao and Luen-Fai Tam, On the heat equation for harmonic maps from noncompact manifolds ........................... 129

John Marafino, Boundary behavior of a conformal mapping . .......... 147

Ji Min, A remark on the symmetry of solutions to nonlinear elliptic

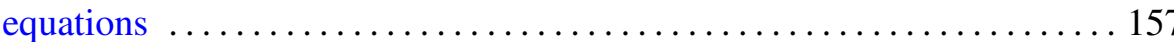

Paul Nevai and Walter Van Assche, Compact perturbations of orthogonal

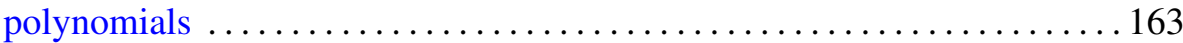

Kyril Tintarev, Level set maxima and quasilinear elliptic problems ....... 185 